\title{
Association of single nucleotide polymorphism Rs2236518 in PRDM16 gene with BMI in Chinese males
}

Hua YUE, Jin-wei HE, Yao-hua KE, Hao ZHANG, Chun WANG, Wei-wei HU, Jie-mei GU, Wen-zhen FU, Yun-qiu HU, Miao LI, Yu-juan LIU, Zhen-lin ZHANG*

Metabolic Bone Disease and Genetic Research Unit, Department of Osteoporosis and Bone Disease, Shanghai Jiao Tong University Affiliated the Sixth People's Hospital, Shanghai 200233, China

Aim: PRD1-BF-1-RIZ1 homologous domain containing protein-16 (PRDM16) is a cell-autonomous transcriptional component that stimulates the development of brown fat cells. The aim of this study was to investigate the contribution of genetic variants of PRDM16 to obesity-related phenotype variations in Chinese.

Methods: A total of 3204 subjects (consisting of 400 male-offspring nuclear families, 401 female-offspring nuclear families, and 729 unrelated older males) were recruited. Ten tag single nucleotide polymorphisms (SNPS) within the PRDM16 gene were genotyped using multiplex quantitative real-time PCR by Taqman assay. Body compositions were measured by dual-energy X-ray absorptiometry (DXA). The associations of the SNPs with the obesity-related phenotypes were analyzed using the quantitative transmission disequilibrium test (QTDT), GLM-ANOVA and PLINK statistical methods.

Results: Rs2236518 was the only SNP that was associated with BMI in young (aged 20-40 years) males ( $P=0.011$ ) using QTDT, and in the older men (aged 50-80 years) $(P=0.003)$ using GLM-ANOVA. No significant associations were detected in the females. Nor was a relationship found between any haplotype and obesity-related phenotypes. When PLINK was used, no significant relationship was detected between 10 SNPs and obesity-related phenotypes in any of the studied cohorts.

Conclusion: Rs2236518 is associated with BMI in the young males (using QTDT), and the older males (using GLM-ANOVA). However, the result is not confirmed using PLINK. The discrepancy needs to be further addressed.

Keywords: PRD1-BF-1-RIZ1 homologous domain containing protein-16 (PRDM16); SNPs; obesity; BMI; brown adipose tissue; quantitative transmission disequilibrium test (QTDT); general linear model-ANOVA (GLM-ANOVA); PLINK; dual-energy X-ray absorptiometry; Chinese male

Acta Pharmacologica Sinica (2013) 34: 710-716; doi: 10.1038/aps.2012.201; published online 25 Mar 2013

\section{Introduction}

Obesity is a state of excess body fat that results from excess energy storage. Human obesity belongs to a group of risk factors known as the metabolic syndrome, which is influenced by genetic, physiological, behavioral, and socio-cultural factors $^{[1,2]}$. Therefore, obesity is currently an area of interest for research. Brown adipose tissue (BAT) dissipates chemical energy to produce heat as a defense against cold. Interest in the development and regulation of BAT has exploded in the past few years. Clearly, much of the interest in this cell type is due to its role in preventing hypothermia and obesity. PRD1-

\footnotetext{
* To whom correspondence should be addressed.

E-mail ZZL2002@medmail.com.cn

Received 2012-10-10 Accepted 2012-12-24
}

BF-1-RIZ1 homologous domain containing protein-16 (PRDM16) is the first known cell-autonomous transcriptional component that is both necessary and sufficient to stimulate the development of brown fat cells ${ }^{[3]}$.

PRDM16 is situated on chromosome $1 \mathrm{p} 36.3^{[4]}$. It is a 140 $\mathrm{kDa}$ zinc-finger protein that was originally identified at a chromosomal breakpoint in $\mathrm{t}(1 ; 3)(\mathrm{p} 36 ; \mathrm{q} 21)$-positive human acute myeloid leukemia cells, and it contains 17 coding exons and spans approximately $400 \mathrm{~kb}^{[5]}$. In 2007 , one study found that brown fat cells contain an abundance of the zinc-finger protein PRDM16 $6^{[6]}$. Soon afterwards, another study demonstrated that PRDM16 can act as a bi-directional switch that directs cell fate to skeletal myoblasts or brown fat cells ${ }^{[7]}$. In 2008, two studies presented evidence that PRDM16 stimulates brown adipogenesis by binding to the peroxisome proliferator-activated 
receptor- $\gamma(P P A R-\gamma)$ and activating its transcriptional function. The loss of PRDM16 from brown fat precursors causes a loss of brown fat characteristics and promotes muscle differentiation. Conversely, the ectopic expression of PRDM16 in myoblasts induces differentiation into brown fat cells ${ }^{[8,9]}$. Animal studies have indicated that PRDM16 transgenic mice display increased energy expenditure, limited weight gain, and improved glucose tolerance in response to a high-fat diet $^{[10]}$. Therefore, the above findings suggest new therapeutic avenues for reducing obesity and its associated diseases. These studies prompted our hypothesis that PRDM16 genetic polymorphisms are associated with variations in body fat mass and lean mass in humans.

In recent years, a wealth of studies have focused on the role of PRDM16 in BAT and white adipose tissue (WAT) and on the possible mechanisms by which PRDM16 directs cell fate to skeletal myoblasts or brown fat cells ${ }^{[9,11-13]}$. To our knowledge, no study has been performed on the genetic association of PRDM16 polymorphisms with obesity-related phenotypic variation in humans. In the present study, based on a large sample including two Chinese nuclear families and one independent cohort, we performed family-based [quantitative transmission disequilibrium test (QTDT)] and populationbased (ANOVA) association studies of the 10 tag single nucleotide polymorphisms (SNPs) in the PRDM16 gene to examine whether these SNPs in PRDM16 contribute to the observed variation in obesity phenotypes in the Chinese Han population. The purpose of this study was to establish an important foundation for the further elucidation of the multiple mechanisms by which PRDM16 modulates obesity phenotypes.

\section{Materials and methods}

\section{Subjects}

All study subjects belonged to the Chinese Han ethnic group. For each study subject, we collected information on age, sex, medical history, family history, marital status, physical activity, alcohol use, dietary habits, and smoking history. We also collected information on menses, obstetrical history, and history of hormonal contraceptive use in the female subjects. Exclusion criteria were the same as in our previous studies ${ }^{[14]}$.

The study was approved by the Ethics Committee of the Shanghai Jiao Tong University Affiliated Sixth People's Hospital. All subjects involved in this study were recruited by an osteoporosis center from a local population in Shanghai City, which is located in the middle of the east coast of China, and all subjects signed written informed consent before entering this study.

\section{Anthropometric measurements}

In the male-offspring nuclear families, fat mass $(\mathrm{kg})$ and lean mass (kg) (including arms, legs, trunk, and total body) were measured by dual-energy X-ray absorptiometry (DXA) on a Lunar Prodigy (GE Lunar Corp, Madison, WI, USA). The DXA scanner was on fan-beam mode. The machine was calibrated daily. Its coefficients of variability $(\mathrm{CV})$ (obtained from 7 volunteers with 5 measurements each) for the fat mass of the upper limbs, lower limbs, trunk, and total body were $3.72 \%$, $3.28 \%, 2.52 \%$, and $3.72 \%$, respectively; the $C V$ for the lean mass measurements at the above sites were $1.18 \%, 1.59 \%, 1.12 \%$, and $1.18 \%$, respectively ${ }^{[14]}$. The long-term precision (expressed as the $C V$ of our DXA instrument that was determined by daily measurements of a phantom) was $0.45 \%$ during the study period ${ }^{[14]}$.

Height was measured to the nearest centimeter on a wallmounted stadiometer, and body weight was measured to the nearest $0.1 \mathrm{~kg}$ on a standard balance beam scale, with subjects wearing light indoor clothing and no shoes. Both the stadiometer and the balance beam scale were regularly calibrated during the study. Body mass index (BMI) was calculated as the weight in kilograms divided by the square of the height in meters, and the fat/lean mass percentage (FM\%/LM\%) was calculated as the ratio of the fat/lean mass to body weight (ie, the sum of fat mass, lean mass, and bone mass).

\section{SNP selection and genotyping}

The SNPs were selected from the National Center for Biotechnology Information (NCBI) dbSNP and HapMap databases. The SNP selection was based on the following criteria: (1) validation status, especially in a Chinese population; (2) the degree of heterozygosity [minor allele frequencies (MAFs)>0.4]; (3) the pairwise linkage disequilibrium (LD) of the algorithmdetermined bin of the SNP exceeding a threshold value $\left(r^{2}=0.8\right)$; and (4) the SNP being a maximally informative site for PRDM16. Therefore, we selected 10 tag SNPs: rs731031, rs12095716, rs4415513, rs11578011, rs12024847, rs2483225, rs4648500, rs870171, rs2493272, and rs2236518. Among these SNPs, only rs2236518 is located in the 3'UTR region of exon 17. The other nine SNPs are all located in introns.

A 5-mL blood sample was drawn from all subjects after a 12-h overnight fast and combined with disodium EDTA. Genomic DNA was extracted from the peripheral blood samples by routine methods. The TaqMan allelic discrimination assay (Applied Biosystems, Foster City, CA, USA) was used for the genotyping. The primer and probe sequences were optimized using the SNP assay-by-design service of Applied Biosystems. The amplification and allelic discrimination were performed in an Mx3000P Real-Time PCR System (Stratagene, CA). Random duplicate genotyping was routinely undertaken during the study and a mean genotyping error rate of less than $1 \%$ was found for all SNPs.

\section{LD and haplotype analyses}

Haplotypes were constructed from the population genotype data using the Stephens algorithm and the Phase program version 2.0.2 $2^{[15]}$. The significance level of the LD between the markers for the PRDM16 gene was assessed using the observed haplotypes, the allele frequencies, and the Haploview software (version 3.2). We examined Lewontin's $D^{\prime}$ and the $r^{2}$ coefficient between all pairs of biallelic loci. The frequencies of the genotypes and haplotypes were calculated using a group of 800 unrelated subjects (the parents from the 400 male-offspring nuclear families). 


\section{Statistical analyses}

The allele frequencies were estimated by gene counting. The Hardy-Weinberg equilibrium was tested by a $\chi^{2}$ goodness-offit statistic. The QTDT program using the orthogonal model was used to test for population stratification, linkage, and within-family association between the SNPs, haplotypes, and obesity-related phenotypes. The QTDT software package is available on the internet (http:/ / www.sph.umich.edu/csg/ abecasis/QTDT/). The obesity phenotypes were adjusted by age. Owing to the possibility of false-positive results in multiple tests, 1000 permutations of the data sets were performed to obtain the empirical $P$ values and assess the reliability of the results. The QTDT program generates $P$ values for various tests using a distribution that is asymptotically $x^{2}$. A $P$ value threshold of 0.05 was considered significant for all of the analyses.

In addition, a general linear model-ANOVA (GLMANOVA) was performed for our independent cohort of 729 older men. This analysis was used to compare the mean values of the phenotypic variables across the genotype combinations while adjusting for covariates (age). The statistical analysis was performed using the SPSS (Statistical Package for the Social Sciences) software package, version 11.0 (SPSS, Chicago, IL, USA).

In this study, PLINK ${ }^{[16]}$ was also used in quality control filtering and association tests. $P$ values $<0.05$ were defined as nominally significant, and samples were further subjected to false discovery rate (FDR) correction to account for multiple comparison problems. A $P$ value $<0.05$ after Bonferroni correction for multiple tests was used to determine statistical significance.

\section{Results}

\section{The basic characteristics of the study subjects}

Four hundred male-offspring nuclear families, consisting of 1215 individuals with at least 1 healthy male child aged 18-44 years (mean age $30.4 \pm 6.1$ ), were recruited in 2004-2007. The average family size was 3.03; 412 families had 1 child and 15 had 2 children. The 401 female-offspring nuclear families, consisting of 1260 individuals with at least 1 healthy female child aged 20-40 years (mean age 31.4 \pm 5.8 ), were recruited in 2000-2004. The average family size was 3.14; 348 families had 1 child, 50 had 2 children, 2 had 3 children, and 1 had 4 children. All recruited daughters were pre-menopausal. An independent study cohort, consisting of 729 unrelated healthy males aged 50-80 years (mean age 61.1 \pm 7.1 ), was recruited in 2008-2009. The basic characteristics of the subjects are shown in Table 1.

\section{Association between obesity-related phenotypes and SNPs and haplotypes in the male-offspring nuclear families}

A total of 1215 subjects were successfully genotyped from the 400 families. All 10 polymorphisms met the expectations of Hardy-Weinberg equilibrium (HWE). Detailed information on the PRDM16 SNPs analyzed in this study and the MAFs in dbSNP is listed in Table 2.

Based on the $D^{\prime}$ values, we found two blocks of substantial LD. Block 1 contained rs11578011, rs12024847, and rs2483225 and had $D^{\prime}$ values $\geq 0.69$, and block 2 contained rs4648500, rs870171, and rs2493272 and had $D^{\prime}$ values $\geq 0.71$ (Figure 1). Owing to the strong LD among the polymorphisms, 7 frequent haplotypes were inferred in block 1 and block 2 using the likelihood method from the PHASE software. The haplotypes and their frequencies in block 1 were GTA (53.8\%), ACG (25.4\%), ATA (6.5\%), ACA (4.8\%), GCG (4.1\%), GCA (3.7\%), and GTG $(1.2 \%)$. The 7 haplotypes in block 1 together accounted for $99.5 \%$ of the total population. The haplotypes and their frequencies in block 2 were GCT $(39.2 \%)$, TAC (34.9\%), TCT $(2.9 \%)$, GCC (6.9\%), TCC (6.3\%), GAC (5.8\%), and TAT $(3.0 \%)$. The 7 haplotypes in block 2 altogether accounted for $99.1 \%$ of the total population.

There were 279, 293, 296, 290, 294, 256,285, 284, 280, and 250 informative nuclear families for the TDT analysis at rs731031, rs12095716, rs4415513, rs11578011, rs12024847, rs2483225, rs4648500, rs870171, rs2493272, and rs2236518, respectively. Population stratification was detected for rs2236518 and trunk fat mass $(P=0.023)$. Rs2236518 had significant within-family associations with BMI $(P=0.011)$. One thousand permutation

Table 1. Basic characteristics of the study cohorts. All data are presented as mean \pm SD for the raw phenotype values without adjustment. FM\% is the abbreviation of fat mass percentage and LM\% is the abbreviation of lean mass percentage.

\begin{tabular}{|c|c|c|c|c|c|c|c|}
\hline & \multicolumn{3}{|c|}{ Male-offspring nuclear families } & \multicolumn{3}{|c|}{ Female-offspring nuclear families } & \multirow{2}{*}{$\begin{array}{l}\text { Independent } \\
\text { cohort } \\
\text { Older males }\end{array}$} \\
\hline & Father & Mother & Son & Father & Mother & Daughter & \\
\hline Number & 400 & 400 & 415 & 401 & 401 & 458 & 729 \\
\hline Age (years) & $61.1 \pm 7.1$ & $58.4 \pm 6.4$ & $30.4 \pm 6.1$ & $62.4 \pm 6.6$ & $59.5 \pm 6.6$ & $31.4 \pm 5.8$ & $61.1 \pm 7.1$ \\
\hline Height $(\mathrm{cm})$ & $166.7 \pm 6.0$ & $155.8 \pm 5.5$ & $173.0 \pm 5.9$ & $166.2 \pm 6.1$ & $154.6 \pm 5.6$ & $159.8 \pm 5.2$ & $166.7 \pm 6.0$ \\
\hline Fat mass (kg) & - & - & $16.31 \pm 7.56$ & - & - & - & - \\
\hline Lean mass (kg) & - & - & $51.43 \pm 5.76$ & - & - & - & - \\
\hline Trunk fat mass (kg) & - & - & $9.34 \pm 4.37$ & - & - & - & - \\
\hline $\mathrm{FM} \%$ & - & - & $21.9 \pm 7.3$ & - & - & - & - \\
\hline LM\% & - & - & $73.2 \pm 6.9$ & - & - & - & - \\
\hline
\end{tabular}


Table 2. Information on the PRDM16 polymorphisms used in this study.

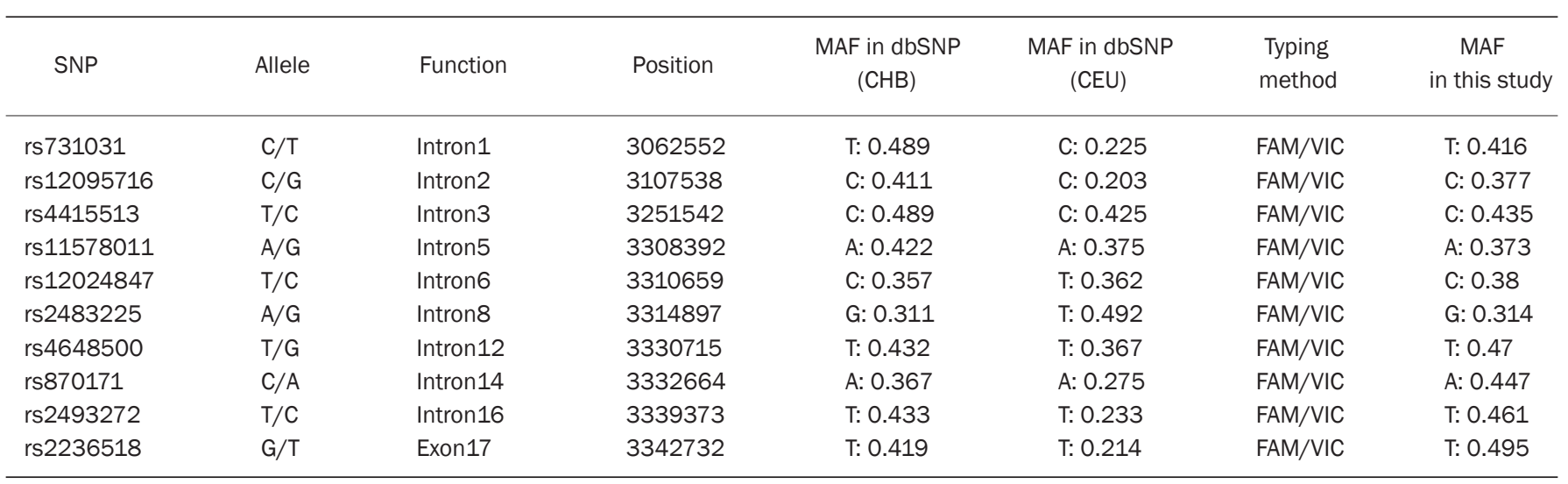

MAF, minor-allele frequency; FAS, fluorescent allele-specific PCR (FAM/VIC).

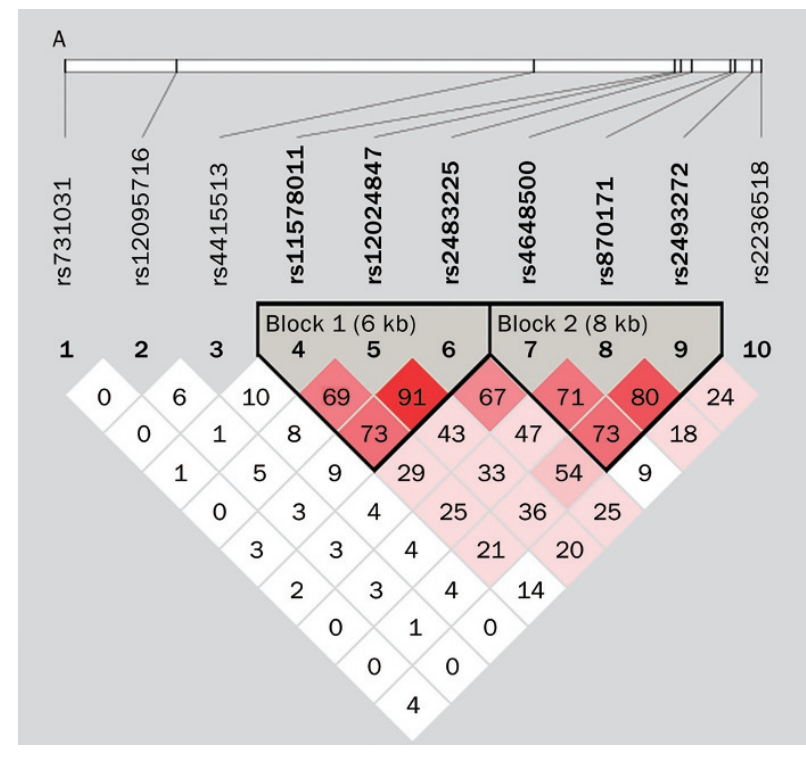

Figure 1. The linkage disequilibrium (LD) pattern in the PRDM16 gene is depicted as an LD matrix. The classic $D^{\prime}$ measurement for all pairs of SNP markers was calculated to construct the LD matrix. The $D^{\prime}$ value of each panel is substituted with different colors. Squares in red indicate a strong LD.

tests were performed to improve fidelity and further confirm the above findings $(P=0.021)$ (Table 3$)$. We failed to find a relationship between any haplotype and obesity-related phenotypes (data not shown).

Rs2236518 was the only polymorphism that showed association with BMI in the male-offspring nuclear families. Therefore, rs2236518 was selected to further analyze in the femaleoffspring nuclear families and the independent cohort consisting of 729 older males.

\section{Association between BMI and rs2236518 in the female-offspring nuclear families}

Because fat mass values had not been measured in females,
BMI was the only obesity-related phenotype whose association with the genotypes could be tested. However, no significant within-family association between rs2236518 and BMI was detected using QTDT in daughters from the female-offspring nuclear families (data not shown).

\section{Association between rs2236518 and BMI in 729 older males}

Only BMI, age, height, and weight had been measured in the unrelated 729 older males when they were recruited, and BMI was the representative obesity-related phenotype in this cohort. The BMI values were adjusted by age as covariates. Rs2236518 was found to be associated with BMI in the ANOVA model $(P=0.003)$. The adjusted BMI was 3.65\% higher in the subjects carrying the TT genotype than in the individuals with the GG genotype; the adjusted BMI was 3.4\% higher in the subjects carrying the GT genotype than in the subjects with the GG genotype. Rs2236518 explained $1.55 \%$ of the BMI variation (Table 4).

\section{Association between rs2236518 and BMI in the three studied cohorts using PLINK}

Unfortunately, no significant relationship between rs2236518 and BMI was detected in any of the three groups (Table 5).

\section{Discussion}

Obesity is a health problem that is caused by disequilibrium between caloric intake and expenditure, and it presents with excessive body fat accumulation. Obesity is a serious public health problem, and individuals with obesity are more likely to develop other serious diseases, such as diabetes, hypertension, and coronary heart disease ${ }^{[17]}$. BMI and fat mass are different obesity-related phenotypes that are known to be under strong genetic control (with a suggested heritability of $40 \%-60 \%)^{[18]}$. Heritability, candidate genes, and genome-wide linkage analyses for fat mass and lean mass have been widely reported in numerous publications ${ }^{[19,20]}$. In this study, we investigated the associations between PRDM16 gene polymorphisms and DXA-determined fat mass in our large samples. 
Table 3. Association between PRDM16 gene polymorphisms and obesity-related phenotypes (using QTDT). Each obesity-related phenotype is adjusted for age. Bold indicates significant $P$ values $(P<0.05)$.

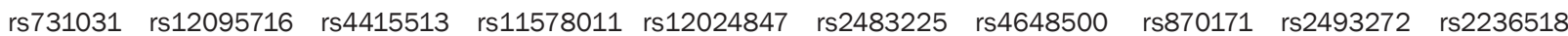

\begin{tabular}{|c|c|c|c|c|c|c|c|c|c|c|}
\hline \multicolumn{11}{|c|}{ Tests of population stratification } \\
\hline BMI & 0.786 & 0.388 & 0.645 & 0.370 & 0.731 & 0.783 & 0.277 & 0.459 & 0.950 & 0.114 \\
\hline runk FM & 0.312 & 1.000 & 0.147 & 0.856 & 1.000 & 1.000 & 0.305 & 1.000 & 0.889 & 0.023 \\
\hline Total FM & 0.179 & 0.338 & 0.106 & 0.724 & 0.743 & 0.595 & 0.296 & 1.000 & 0.640 & 0.270 \\
\hline \multicolumn{11}{|c|}{ Tests of total association } \\
\hline BMI & 0.911 & 0.359 & 0.180 & 0.308 & 0.028 & 0.019 & 0.775 & 0.761 & 0.642 & 0.007 \\
\hline Total FM & 0.206 & 0.811 & 0.966 & 0.518 & 0.559 & 0.963 & 0.289 & 0.469 & 0.307 & 0.004 \\
\hline PFM & 0.262 & 0.875 & 0.702 & 0.951 & 0.991 & 0.694 & 0.331 & 0.530 & 0.529 & 0.394 \\
\hline \multicolumn{11}{|c|}{ Tests of within-family association } \\
\hline BMI & 0.850 & 0.751 & 0.284 & 0.201 & 0.440 & 0.197 & 0.274 & 0.167 & 0.870 & 0.011 \\
\hline Trunk FM & 0.866 & 0.076 & 0.413 & 0.794 & 0.056 & 1.000 & 1.000 & 0.707 & 0.442 & 0.060 \\
\hline \multicolumn{11}{|c|}{1000 p 1000 permutations of within-family association } \\
\hline BMI & 0.829 & 0.770 & 0.345 & 0.226 & 0.481 & 0.219 & 0.303 & 0.632 & 0.895 & 0.021 \\
\hline
\end{tabular}

Table 4. Association between rs2236518 and BMI in the the unrelated older males using GLM-ANOVA. Values are given as means \pm SD; $P$ values for ANOVA (adjusted for age). ${ }^{\mathrm{c}} P=0.002, \mathrm{G} / \mathrm{G}$ versus $\mathrm{T} / \mathrm{T} ;{ }^{\mathrm{f}} P=0.006, \mathrm{G} / \mathrm{G}$ versus $\mathrm{G} / \mathrm{T}$.

\begin{tabular}{lll}
\hline Genotype & $n$ & \multicolumn{1}{c}{ BMI } \\
\hline Rs2236518 & & \\
$G / G$ & 207 & $24.089 \pm 3.141^{\text {cf }}$ \\
$G / T$ & 217 & $24.911 \pm 3.003$ \\
T/T & 305 & $24.967 \pm 3.093$ \\
$P$ value & & 0.003 \\
\hline
\end{tabular}

Table 5. Association between $\mathrm{rs} 2236518$ and BMI in the three studied cohorts using PLINK.

\begin{tabular}{lccc}
\hline \multicolumn{3}{c}{ SNP } & \multicolumn{3}{c}{ BMI } \\
\hline Rs2236518 & Young males & Young females & Unrelated older males \\
Bonferoni $P$ & 0.1883 & 0.6435 & 0.5613 \\
FDR $P$ & 0.0779 & 0.4755 & 0.3926 \\
\hline
\end{tabular}

FDR, false discovery rate; BMI, body mass index. A $P$ value $<0.05$ after Bonferroni correction for multiple tests was used to determine statistical significance.

BMI and fat mass were chosen as the obesity-related phenotypes in our study for two reasons. First, the World Health Organization (WHO) has proposed BMI as a simple and practical measure of obesity. In addition, BMI is the most commonly used obesity phenotype in epidemiological studies. Second, fat mass can be easily and accurately determined by DXA, and it can be used to confirm BMI findings, given that BMI alone may not always be appropriate for defining obesity $^{[21]}$.

To eliminate false positive results, QTDT was used to detect population stratification and analyze within-family associations, and within-family association between rs2236518 and BMI $(P=0.011)$ was detected in the male-offspring nuclear families. However, the haplotype analysis did not provide any significant evidence to support the above positive result. Interestingly, the relationship between rs2236518 and BMI was detected in the independent cohort of 729 older males using GLM-ANOVA, whereas no association was found between rs2236518 and BMI in the daughters from the female-offspring nuclear families. This discrepancy suggests that the association between PRDM16 and BMI variations may be gender specific. The latest studies also supported this hypothesis that the association between PRDM16 genotype and BMI is specific to females. Xi et al ${ }^{[22]}$ confirmed the synthetic effect of SNPs on the indices of adiposity and risk of obesity in Chinese girls but not in Chinese boys. Another study performed in Chinese children also indicated that gender differences may exist in various obesity-related genes ${ }^{[23]}$. In our study, the reasons for the difference between the sexes may be as follows: 1) the adipose tissue distribution between women and men is quite different and 2) bone geometric structures vary between genders. Nevertheless, studies with larger sample sizes are needed to confirm these hypotheses. However, it is difficult to explain 
why no significant relationship between 10 SNPs and obesityrelated phenotypes was detected in any of the studied cohorts by using PLINK, which is quite different from the results we obtained using other statistical methods. Hence, we cannot draw a clear conclusion regarding whether rs2236518 is associated with the normal variation in obesity-related phenotypes in Chinese people. Further work should be performed to further verify the results we obtained.

In recent years, a large number of studies have examined the mechanisms of obesity, especially brown adipogenesis. One recent study showed that Plac8 is a critical upstream regulator of brown fat differentiation, and its overexpression induces $C / E B P \beta$ and PRDM16 expression using brown preadipocytes $^{[24]}$. Cao et al found that environmental enrichment induced the upregulation of PRDM16 in white fat, whereas hypothalamic overexpression of brain-derived neurotrophic factor reproduced the enrichment-associated activation of the brown fat gene program and lean phenotype ${ }^{[24]}$. In addition, Yadav et al showed that $S \operatorname{mad} 3^{(-/-)}$adipocytes demonstrated a marked increase in mitochondrial biogenesis, with a corresponding increase in basal respiration, and that Smad3 acted as a repressor of PGC-1a expression and regulated energy homeostasis ${ }^{[25]}$. One recent animal study indicated that the WAT of FSP27-deficient mice had a gene expression profile similar to that of BAT, and that FSP27 acted as a molecular determinant that controls $C / E B P a / \beta$ and PRDM16 gene expression and components of the CAMP signaling pathway ${ }^{[26]}$. Although experiments in vitro or in vivo have demonstrated the close relationship between PRDM16 and obesity, our study does not confirm the link in humans.

Our study has several important strengths. First, our subjects were taken from 400 male-offspring nuclear families, 401 female-offspring nuclear families, and 729 independent older males, which make our study more credible, and the sample size was large enough to detect quantitative trait loci (QTL). Second, we investigated 10 PRDM16 tag SNPs. The tag SNPS included more informative SNPs, and they may have been more representative than non-tag SNPs. Third, the tag SNPs we selected had relatively high heterozygosity, to maximize the study's power to detect associations with obesity phenotypes.

Our study also has some limitations. First, we studied only healthy men and healthy young females of the Chinese Han ethnic group, so caution is needed when generalizing our conclusions to other populations. Second, because fat mass and lean mass values were not measured in the female-offspring nuclear families and the independent cohort of 729 older males, the only obesity-related phenotype studied in these two populations was BMI, which may not always be appropriate for defining obesity. Thus, our analysis of the relationship between PRDM16 genetic variations and obesity-related phenotypes was not complete. Additional obesity phenotypes are needed for further analysis.

To our knowledge, this is the first study to investigate the relationship between PRDM16 SNPs and haplotypes and obesity-related phenotypes in the Chinese Han population. Our study suggested that genetic variation at the PRDM16 gene may not contribute to the obesity-related phenotypic variability in Chinese Han population. There is not enough evidence to show that the PRDM16 gene is a candidate gene for the genetic determination of body composition in the Chinese Han ethnic group. Larger sample sizes and samples from different ethnic populations are needed to replicate our results.

\section{Acknowledgements}

The study was supported by grants from the National Natural Science Foundation of China (№ 81170803, 81070692, 81000360, and 30800387), Shanghai Rising-Star Program (№ 11QA1404900), Shanghai Natural Science Foundation (№ 11ZR1427300), and Academic Leaders in Health Sciences in Shanghai (№ XBR2011014).

\section{Author contribution}

Hua YUE genotyped SNPs, conducted statistical analyses and drafted the manuscript. Zhen-lin ZHANG conceived and designed the study and revised the manuscript. Jin-wei HE guided the work of the genetic laboratory and guaranteed and confirmed the quality of the genetic data. Yao-hua KE, Hao ZHANG, Chun WANG, Wei-wei HU, and Jie-mei GU were involved in the recruitment of the nuclear families. Wenzhen FU and Yu-juan LIU contributed to collection of blood specimens and DNA database management. Yun-qiu HU and Miao LI were responsible for measuring body composition. All authors read and approved the final manuscript.

\section{References}

1 Perusse L, Bouchard C. Genotype-environment interaction in human obesity. Nutr Rev 1999; 57: S31-S37.

2 Zhang ZY, Wang MW. Obesity, a health burden of a global nature. Acta Pharmacol Sin 2012; 33: 145-7.

3 Kajimura S, Seale P, Spiegelman BM. Transcriptional control of brown fat development. Cell Metab 2010; 11: 257-62.

4 Mochizuki N, Shimizu S, Nagasawa T, Tanaka H, Taniwaki M, Yokota J, et al. A novel gene, MEL1, mapped to 1p36.3 is highly homologous to the MDS1/EVI1 gene and is transcriptionally activated in $t(1 ; 3)$ (p36;q21)-positive leukemia cells. Blood 2000; 96: 3209-14.

5 Nishikata I, Sasaki H, Iga M, Tateno Y, Imayoshi S, Asou N, et al. A novel EVI1 gene family, MEL1, lacking a PR domain (MELIS) is expressed mainly in $\mathrm{t}(1 ; 3)(\mathrm{p} 36$; $\mathrm{q} 21)$ - positive $\mathrm{AML}$ and blocks G-CSF-induced myeloid differentiation. Blood 2003; 102: 3323-32.

6 Seale P, Kajimura S, Yang W, Chin S, Rohas LM, Uldry M, et al. Transcriptional control of brown fat determination by PRDM16. Cell Metab 2007; 6: 38-54.

7 Frühbeck G, Sesma P, Burrell MA. PRDM16: the interconvertible adipo-myocyte switch. Trends Cell Biol 2009; 19: 141-6.

8 Tseng YH, Kokkotou E, Schulz TJ, Huang TL, Winnay JN, Taniguchi $\mathrm{CM}$, et al. New role of bone morphogenetic protein 7 in brown adipogenesis and energy expenditure. Nature 2008; 454: 1000-4.

9 Seale P, Bjork B, Yang W, Kajimura S, Chin S, Kuang S, et al. PRDM16 controls a brown fat/skeletal muscle switch. Nature 2008; 454: 961-7.

10 Seale P, Conroe HM, Estall J, Kajimura S, Frontini A, Ishibashi J, et al. Prdm16 determines the thermogenic program of subcutaneous white adipose tissue in mice. J Clin Invest 2011; 121: 96-105. 
11 Kajimura S, Seale P, Kubota K, Lunsford E, Frangioni JV, Gygi SP, et al. Initiation of myoblast to brown fat switch by a PRDM16-C/EBP-beta transcriptional complex. Nature 2009; 460: 1154-8.

12 Kajimura S, Seale P, Tomaru T, Erdjument-Bromage H, Cooper MP, Ruas JL, et al. Regulation of the brown and white fat gene programs through a PRDM16/CtBP transcriptional complex. Genes Dev 2008; 22: $1397-409$.

13 Karamitri A, Shore AM, Docherty K, Speakman JR, Lomax MA. Combinatorial transcription factor regulation of the cyclic AMP-response element on the Pgc-1alpha promoter in white 3T3-L1 and brown HIB1B preadipocytes. J Biol Chem 2009; 284: 20738-52.

14 Xiao WJ, He JW, Zhang H, Hu WW, Gu JM, Yue H, et al. ALOX12 polymorphisms are associated with fat mass but not peak bone mineral density in Chinese nuclear families. Int J Obes (Lond) 2011; 35 : 378-86.

15 Stephens M, Smith NJ, Donnelly P. A new statistical method for haplotype reconstruction from population data. Am J Hum Genet 2001; 68: 978-89.

16 Purcell S, Neale B, Todd-Brown K, Thomas L, Ferreira MA, Bender D,Maller J, Sklar P, de Bakker PI, Daly MJ, Sham PC. PLINK: a tool set for whole-genome association and population-based linkage analyses. Am J Hum Genet 2007; 81: 559-75.

17 Mokdad AH, Ford ES, Bowman BA, Dietz WH, Vinicor F, Bales VS, et al. Prevalence of obesity, diabetes, and obesity-related health risk factors. JAMA 2003; 289: 76-9.

18 Laclaustra M, Corella D, Ordovas JM. Metabolic syndrome pathophysiology: the role of adipose tissue. Nutr Metab Cardiovasc Dis 2007; 17: 125-39.

19 Livshits G, Kato BS, Wilson SG, Spector TD. Linkage of gene to total lean body mass in normal women. J Clin Endocrinol Metab 2007; 92: 3171-6.

20 Liu YZ, Pei YF, Liu JF, Yang F, Guo Y, Zhang L, et al. Powerful bivariate genome-wide association analyses suggest the SOX 6 gene influencing both obesity and osteoporosis phenotypes in males. PLoS One 2009; 4: e6827.

21 Jimenez-Preitner M, Berney X, Uldry M, Vitali A, Cinti S, Ledford JG, et al. Plac8 is an inducer of $C / E B P \beta$ required for brown fat differentiation, thermoregulation, and control of body weight. Cell Metab 2011; 14: 658-70.

22 Xi B, Shen Y, Reilly KH, Zhao X, Cheng H, Hou D, et al. Sex-dependent associations of genetic variants identified by GWAS with indices of adiposity and obesity risk in a Chinese children population. Clin Endocrinol (Oxf) 2012 Nov 3. doi: 10.1111/cen.12091. [Epub ahead of print]

23 Wang J, Mei H, Chen W, Jiang Y, Sun W, Li F, et al. Study of eight GWAS-identified common variants for association with obesity-related indices in Chinese children at puberty. Int J Obes (Lond) 2012; 36: 542-7.

24 Cao L, Choi EY, Liu X, Martin A, Wang C, Xu X, et al. White to brown fat phenotypic switch induced by genetic and environmental activation of a hypothalamic-adipocyte axis. Cell Metab 2011; 14: 324-38.

25 Yadav H, Quijano C, Kamaraju AK, Gavrilova O, Malek R, Chen W, et al. Protection from obesity and diabetes by blockade of TGF- $\beta / S m a d 3$ signaling. Cell Metab 2011; 14: 67-79.

26 Li D, Zhang Y, Xu L, Zhou L, Wang Y, Xue B, et al. Regulation of gene expression by FSP27 in white and brown adipose tissue. BMC Genomics 2010; 11: 446. 